\title{
In memoriam Mildred Scheel
}

Ihr Name war Programm. Es machte sie innerhalb kürzester Zeit zur populärsten Ärztin Deutschlands. Wo immer sie auftrat, um selbstbewußt, überzeugend und optimistisch für die Krebsbekämpfung zu werben, erblickte man in ihr die entschlossene Kämpferin, die sich unkonventionell und spontan für die Belange krebskranker Mitbürger einsetzte.

Ihr leidenschaftlicher Wunsch, helfen zu wollen, war früh angelegt. 1932 als Tochter des Facharztes für Röntgenologie und Strahlenheilkunde Dr. med. Hans-Hubert Wirtz in Köln geboren, fühlte sie sich bereits in ihrer Jugend zum Arztberuf hingezogen. Geradlinig, wie es ihrem Wesen entsprach, nahm sie nach dem Abitur in Amberg in der bayerischen Landeshauptstadt das Medizinstudium auf und promovierte 1956 an der Chirurgischen Universitätsklinik München über die Symptomatologie und Behandlung des Kardia-karzinoms. Anschließend arbeitete sie zwei Jahre lang als Medizinalassistentin, dann als Assistenzärztin an den Münchner Universitätskliniken. Nach der Approbation ließ sie sich zur Ärztin für Röntgenologie ausbilden.

Ihre Heirat mit Walter Scheel veränderte ihren ursprünglich geplanten Lebensweg als niedergelassene Ärztin grundlegend. 1969 zog das Ehepaar Scheel nach Bonn, wo Walter Scheel das Amt des Außen-ministers, 1974 das des Bundespräsidenten übernahm. Nunmehr «First Lady», gründete Mildred Scheel am 25. September 1974 die DEUTSCHE KREBSHILFE e.V., die zu ihrer Lebensaufgabe wurde. Damit verwirklichte sie, inzwischen Mutter zweier Töchter und eines bolivianischen Adoptivsohnes, einen langgehegten Wunsch aus ihrer Studienzeit: sich gezielt der Krebsbekämpfung zuzuwenden. Mit der Gründung der DEUTSCHEN KREBSHILFE bot Mildred Scheel erstmals jedem Bundesbürger die Möglichkeit an, sich persönlich durch Spenden an der Lösung des Krebsproblems zu beteiligen. Damit definierte sie das bisher vorwiegend der Ärzteschaft vorbehaltene Thema «Krebs» ' auf einer bürgernahen Basis neu. Um die von ihr gewünschte Breitenwirkung zu erzielen, popularisierte sie den Kampf gegen den Krebs über die Medien und machte dadurch die DEUTSCHE KREBSHILFE zur ersten und erfolgreich-sten deutschen Bürgerinitiative gegen die Krebskrankheiten. Je mehr Menschen sich in den folgenden Jahren mit ihrem Lebenswerk identifizierten, desto mehr wurde ihr Name zum Synonym für Krebsbekämpfung. Gerade aber diese Identität von Person und Werk wurde der Schlüssel zum Erfolg der DEUTSCHEN KREBSHILFE.

Programmatisch deckten die Projekte der DEUTSCHEN KREBSHILFE modellhaft alle Bereiche der Krebsvorsorge, Krebsdiagnose und Krebstherapie, der Krebsnachsorge, Krebsforschung sowie der Information und Aufklärung über Krebserkrankungen ab. Ziel war es, neue, richtungsweisende Impulse zu geben, um damit die Entwicklung der Onkologie zu beschleunigen. Dementsprechend besitzen die von der DEUTSCHEN KREBSHILFE geförderten Projekte innovativen Charakter oder dienen dazu, einen sonst nicht zu behebenden akuten Notstand zu beseitigen. Hatten sich die Programme - wie etwa Tumorzentren oder Nachsorgeeinrichtungen - als erfolgreich erwiesen, drängte Mildred Scheel den Staat, diese Projekte zu übernehmen und sie auf breiter Basis flächendeckend einzusetzen. 
Was Mildred Scheel mit diesem Konzept erreichte, veränderte das Bild der bis dahin in der Bundesrepublik praktizierten Krebsbekämpfung von Grund auf. Untrennbar mit ihrem Namen verbunden sind so die Gründung der ersten deutschen Tumorzentren, der Aufbau eines Krebsnachsorgenetzes vor allem im psycho-sozialen Bereich, die Schaffung eines Härtefonds zur unmittelbaren Unterstützung von Krebskran-ken, die durch ihr Leiden in finanzielle Not gerieten, sowie die Intensivierung der Krebsforschung über die Dr.-Mildred-ScheelStiftung/Deutsche Stiftung für Krebsforschung.

Die Strukturen, die sie etwa durch den Aufbau von Tumorzentren, Zentren zur Behandlung von Krebserkrankungen im Kindesalter oder durch die Errichtung von Nachsorgeeinrichtungen schuf, sind heute fester Bestandteil des medizinisch-onkologischen Versorgungsnetzes in der Bundesrepublik. Gleiches gilt für damals noch neuartige Diagnoseverfahren, wie etwa Mammographie oder Computer-tomographie, deren Einführung sie gegen Kritik und Widerstand beschleunigte und durchsetzte. Ähnlich große Verdienste erwarb sie sich nicht zuletzt durch die ständige Propagierung der Krebsfrüherkennungs-untersuchungen, die sie für alternativlos und unerläßlich hielt.

Der Erfolg, den Mildred Scheel mit ihrer DEUTSCHEN KREBSHILFE in der Bevölkerung erzielte, läßt sich messen: Bis zu ihrem Tode vertrauten ihr Burger aus alien Teilen der Bevölkerung 250 Millionen Mark zur Krebsbekämpfung an. Damit wurden mehr als 250 Einzelprogramme finanziert, die sämtlich darauf abzielten, Krebskranken unmittelbar bei der Erkennung, Behandlung und Überwindung ihres Leidens zu helfen. Im Rückblick erweist sich damit ihre Entscheidung, die Krebsbekämpfung in der Bundesrepublik zu enttabuisieren und dadurch den Kampf gegen den Krebs zum persönlichen Anliegen aller Burger zu machen, als historischer Meilenstein in der Bekämpfung der Krebskrankheiten, der ihren Tod am 13. Mai 1985 überdauern wird. 